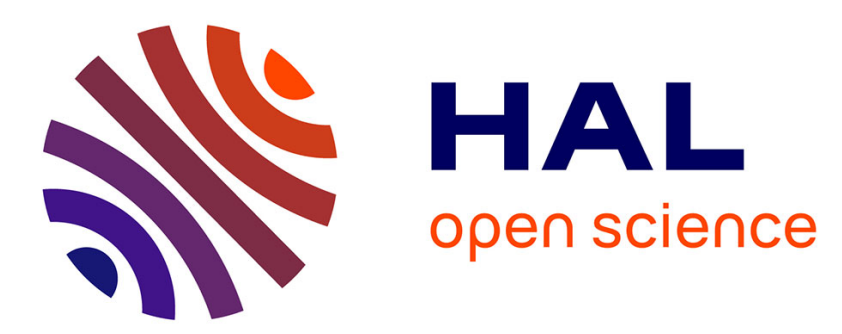

\title{
Modelling of the photometric balance for two-wavelength spatially multiplexed digital holography
}

\author{
Matthieu Piniard, Béatrice Sorrente, Gilles Hug, Pascal Picart
}

\section{To cite this version:}

Matthieu Piniard, Béatrice Sorrente, Gilles Hug, Pascal Picart. Modelling of the photometric balance for two-wavelength spatially multiplexed digital holography. SPIE Optical Metrology 2021, Jun 2021, Online, United States. pp.1178308, 10.1117/12.2593719 . hal-03324271

\section{HAL Id: hal-03324271 \\ https://hal.science/hal-03324271}

Submitted on 23 Aug 2021

HAL is a multi-disciplinary open access archive for the deposit and dissemination of scientific research documents, whether they are published or not. The documents may come from teaching and research institutions in France or abroad, or from public or private research centers.
L'archive ouverte pluridisciplinaire HAL, est destinée au dépôt et à la diffusion de documents scientifiques de niveau recherche, publiés ou non, émanant des établissements d'enseignement et de recherche français ou étrangers, des laboratoires publics ou privés. 


\title{
Modelling of the photometric balance for two-wavelength spatially multiplexed digital holography
}

\author{
Matthieu Piniard $^{\mathrm{a}}$, Béatrice Sorrente ${ }^{\mathrm{a}}$, Gilles Hug ${ }^{\mathrm{b}}$, and Pascal Picart ${ }^{\mathrm{c}}$ \\ ${ }^{a}$ DOTA, ONERA, Université Paris Saclay, 91123 Palaiseau, France \\ ${ }^{b}$ Laboratoire d'Étude des Microstructures, ONERA-CNRS, UMR 104, 29 Avenue de la \\ Division Leclerc, 92320 Châtillon, France \\ 'Laboratoire d'Acoustique de l'Université du Mans (LAUM), UMR 6613, Institut d'Acoustique \\ - Graduate School (IA-GS), CNRS, Le Mans Université, Avenue Olivier Messiaen, 72085 Le \\ Mans, France
}

\begin{abstract}
Multi-wavelength digital holography is a very powerful approach for surface shape measurements. It has the advantage of being contact-less, non-intrusive, and yields full-field surface shape data without any requirement for scanning. This paper proposes the analysis of the standard deviation of noise in surface-shape data from twowavelength spatially-multiplexed digital holograms. The influence of noise on the measurements of the surface shape is described by an analytical approach. Numerical simulations with realistic experimental parameters are provided and discussed.
\end{abstract}

Keywords: digital holography, multi wavelength holography, noise, speckle, speckle decorrelation

\section{INTRODUCTION}

Multi-wavelength digital holography has demonstrated to be a relevant tool for desensitized testing of steep optical surfaces (aspheric mirrors and lenses), ${ }^{1}$ large deformation of structures ${ }^{2}$ or also surface shape profiling. ${ }^{3}$ Such an approach can also be used for surface roughness measurements when the roughness is large compared to the wavelength. ${ }^{4}$ With the advent of digital holography, ${ }^{5}$ a wide range of applications of multi-wavelength holography was demonstrated, such as endoscopic imaging, ${ }^{6,7}$ calibration of mechanical structures, ${ }^{8}$ erosion measurements, ${ }^{9}$ or also in-line industrial inspection. ${ }^{10}$ When dealing with off-axis digital holography and spatial multiplexing of two-wavelength digital holograms, the method becomes real-time, in the sense that the surface shape can be measured at each time instant at which the holograms are recorded. However, due to the roughness of the inspected surface, speckle decorrelation occurs and noise is included in the final data. The noise amount in the data must be investigated in order to define the best processing approach for holograms. This paper proposes the theoretical analysis of the standard deviation of noise in the surface-height data from two-wavelength spatially-multiplexed digital holograms. The influence of noise on the measurements of the surface shape is described by an analytical approach.

\section{TWO-WAVELENGTH DIGITAL HOLOGRAPHY}

Two-wavelength digital holography has many advantages over single wavelength holography. With a unique wavelength, the measured surface height of any natural object (non polished) is ambiguous when larger than the wavelength. Since the surface of the object also includes roughness, then the surface shape cannot be reconstructed because the phase extracted from the digital holograms results from a fully developed speckle pattern. The ambiguity and randomness of this phase can be mitigated with the use of another/several wavelengths leading to what is known as the synthetic wavelength. It follows that the unambiguous range becomes increased from microns to millimeter or larger. Considering two wavelengths $\lambda_{1}$ and $\lambda_{2}$, the synthetic wavelength is given

e-mail: pascal.picart@univ-lemans.fr 
by $\Lambda=\lambda_{1} \lambda_{2} /\left|\lambda_{1}-\lambda_{2}\right| \cdot{ }^{11}$ However, there is an inherent issue of using several wavelengths that is related to the speckle decorrelation arising when the primary illumination wavelength is changed to extend to the next one. This induces a phase noise in the phase difference calculated from the two phases at the two wavelengths. The surface height of the object, $h(x, y)$, is calculated with the following equation which includes the speckle decorrelation noise (here is considered that illumination and observation of the surface are at normal incidence):

$$
h(x, y)=\frac{\Lambda}{4 \pi}\left[\varphi_{2}(x, y)-\varphi_{1}(x, y)+\epsilon_{\text {speckle }}\right],
$$

with $\varphi_{1}, \varphi_{2}$ the optical phases from the two holograms at the two wavelengths and $\epsilon_{\text {speckle }}$ the phase noise due to the speckle decorrelation. It follows that the standard deviation of the surface height is given by:

$$
\sigma_{h}=\frac{\Lambda}{4 \pi} \sqrt{\sigma_{\Delta \varphi}^{2}+\sigma_{\epsilon}^{2}}
$$

with $\sigma_{\Delta \varphi}^{2}=\sigma_{\varphi_{1}}^{2}+\sigma_{\varphi_{2}}^{2}$. The standard deviation of the decorrelation noise depends on the modulus of the complex coherence factor between the two speckle fields at the two wavelengths. ${ }^{12}$ An approximated formula for $\sigma_{\epsilon}$ valid for $|\boldsymbol{\mu}| \in[0.7 ; 1]$ was provided by Picart et al: ${ }^{13}$

$$
\sigma_{\epsilon}=\frac{7}{4}(1-|\boldsymbol{\mu}|)^{\frac{2}{5}}
$$

Thus one obtains,

$$
\sigma_{h}=\frac{\Lambda}{4 \pi} \sqrt{\sigma_{\Delta \varphi}^{2}+\left(\frac{7}{4}\right)^{2}(1-|\boldsymbol{\mu}|)^{\frac{4}{5}}} .
$$

For two-wavelength speckle decorrelation, the modulus of the complex coherence factor depends on the synthetic wavelength and on the standard deviation of the surface roughness, $S_{q}$, so that we have $|\boldsymbol{\mu}|=$ $\exp \left(-\frac{8 \pi^{2} S_{q}^{2}}{\Lambda^{2}}\right) \cdot{ }^{12,14,15}$

\section{ESTIMATION OF THE PHASE NOISE}

Digital holography suffers from the influence of speckle noise, photon noise, quantization noise and electronic noise (readout noise) of the sensor. The influence of these sources of noise must be considered with regard to the dynamics of encoding of the digital hologram. The photon noise $\left(\sigma_{p h}\right)$, quantization noise $\left(\sigma_{q}\right)$ and electronic noise $\left(\sigma_{R o N}\right)$ of the sensor refers here to the technical noise. Additional noise source is found in the dark current noise $\left(\sigma_{D C}\right)$ and background noise $\left(\sigma_{b g}\right)$. The standard deviation of the phase extracted from any off-axis digital hologram can be estimated according to: ${ }^{16}$

$$
\sigma_{\varphi_{i}}=\frac{p_{x} R_{u} \sqrt{2 \pi}}{m_{i} \alpha_{i} N_{s a t}} \sqrt{\sigma_{p h}^{2}+\sigma_{q}^{2}+\sigma_{R o N}^{2}+\sigma_{b g}^{2}+\sigma_{D C}^{2}}
$$

In Eq. (5), $p_{x}$ is the pixel pitch of the sensor, $N_{s a t}$ is the maximum number of photo electrons in pixels at saturation, $m_{i}$ is the modulation of the hologram $\left(m_{i} \leq 1\right), \alpha_{i}$ is the saturation ratio of the hologram $\left(\alpha_{i} \leq 0.25\right)$ and $R_{u}$ is the radius of the spectral bandwidth of the digital hologram. Figure 1(a) depicts the basic scheme for image-plane digital holography for surface-shape measurements. Figure 1(b) illustrates the spectral distribution in the Fourier domain of the digital hologram in spatially multiplexed off-axis two-wavelength holography. The useful +1 order has a spectral spatial bandwidth with diameter $2 R_{u}$, and the extraction of the order is performed by the binary spectral filter having the same bandwidth.

In the case where the exposure time of the digital holograms is small (typ. $<1 \mathrm{~ms})$ and the measured surface is at ambient temperature (typ. $300 \mathrm{~K})$, then the contributions of the dark current noise $\left(\sigma_{D C}\right)$ and background noise $\left(\sigma_{b g}\right)$ are irrelevant and can be neglected in Eq. (5). Whereas $\sigma_{R o N}$ is technical data from the camera provider, 

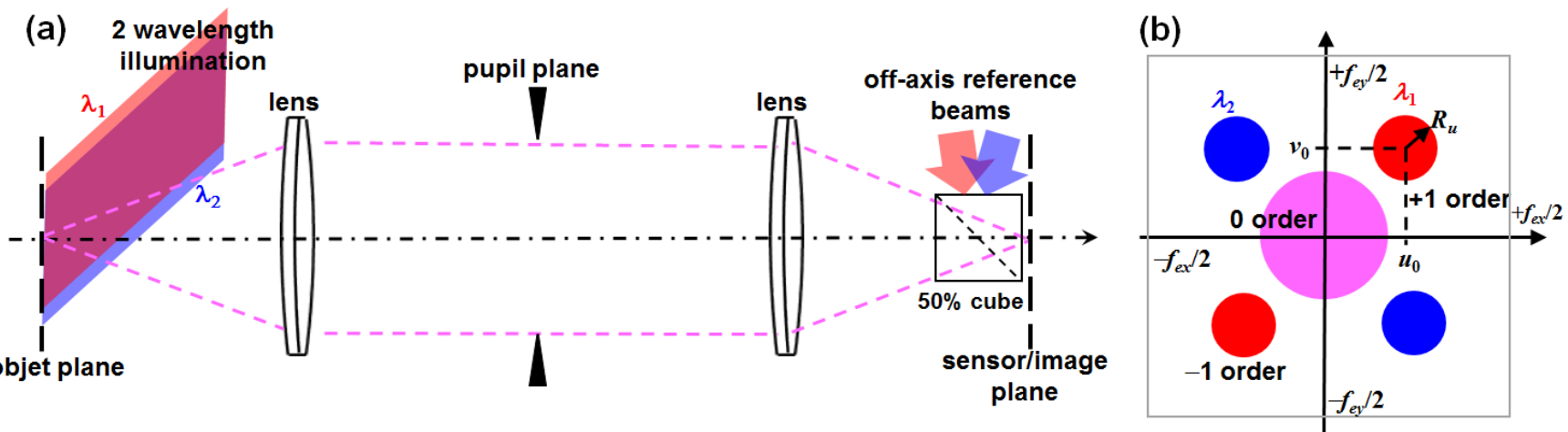

Figure 1. (a) Imaging surface shape through an off-axis digital holographic system, (b) Spectral distribution in the Fourier domain of the off-axis digital holograms.

the photons noise is given by $\sigma_{p h}^{2}=\alpha_{i} N_{\text {sat }}$ and the quantization noise is given by $\sigma_{q}^{2}=N_{\text {sat }}^{2} /\left(12\left(2^{\text {nbits }}-1\right)^{2}\right)$ with nbits the number of quantization bits of the sensor. It follows that we have:

$$
\sigma_{\Delta \varphi}=\frac{p_{x} R_{u} \sqrt{2 \pi}}{N_{\text {sat }} \sqrt{m_{1}^{2} m_{2}^{2} \alpha_{1}^{2} \alpha_{2}^{2}}} \sqrt{N_{\text {sat }}\left(m_{2}^{2} \alpha_{2}^{2} \alpha_{1}+m_{1}^{2} \alpha_{1}^{2} \alpha_{2}\right)+\left(m_{1}^{2} \alpha_{1}^{2}+m_{2}^{2} \alpha_{2}^{2}\right)\left(\frac{N_{\text {sat }}}{\sqrt{12}\left(2^{\text {nbits }}-1\right)}\right)^{2}+\sigma_{\text {RoN }}^{2}} .
$$

In the following, for the sake of simplicity, we consider the ideal case with $m_{1}=m_{2}=m$ and $\alpha_{1}=\alpha_{2}=\alpha$, for which Eq. (6) reduces to:

$$
\sigma_{\Delta \varphi}=\frac{2 p_{x} R_{u} \sqrt{\pi}}{m \alpha N_{\text {sat }}} \sqrt{\alpha N_{\text {sat }}+\left(\frac{N_{\text {sat }}}{\sqrt{12}\left(2^{\text {nbits }}-1\right)}\right)^{2}+\sigma_{\text {RoN }}^{2}} .
$$
to:

By combining Eq. (4) and Eq. (7) one gets the standard deviation of the measured surface height, according

$$
\sigma_{h}=\frac{\Lambda}{4 \pi} \sqrt{\frac{4 \pi p_{x}^{2} R_{u}^{2}}{N_{\text {sat }}^{2} m^{2} \alpha^{2}}\left(\alpha N_{\text {sat }}+\left(\frac{N_{\text {sat }}}{\sqrt{12}\left(2^{\text {nbits }}-1\right)}\right)^{2}+\sigma_{R o N}^{2}\right)+\left(\frac{7}{4}\right)^{2}\left(1-\exp \left(-8 \pi^{2} \frac{S_{q}^{2}}{\Lambda^{2}}\right)\right)^{\frac{4}{5}}} .
$$

Equation (8) is the standard deviation of the surface height computed at any pixel as the product of the synthetic wavelength and the quadratic sum of the technical noise and decorrelation noise. The latter can be calculated from statistics either on the whole field of view or on a restricted number of data points around any considered pixel. For a given synthetic wavelength, the decorrelation noise depends on the value of $S_{q}$ which is also calculated statistically. If the roughness is statistically stationary, then $\sigma_{h}$ is constant over the field of view. If the roughness is not stationary, then the standard deviation locally varies according to $S_{q}$. The uncertainty of the measurement will therefore be higher in the roughest sub-areas.

\section{RESULTS}

Equation (8) can be plotted by considering realistic values of the experimental parameters. For the sensor, one considers $\sigma_{R o N}=6 \mathrm{e}^{-}$, dark current at $\mathrm{DC}(\mathrm{T}=233 \mathrm{~K})=5.10^{-4} \mathrm{e}^{-} / \mathrm{pixel} / \mathrm{s}, N_{\text {sat }}=18000 \mathrm{e}^{-}, p_{x}=6.45 \mu \mathrm{m}$, $R_{u}$ at almost $1 / 4 p_{x} \approx 39 \mathrm{~mm}^{-1}$, and $n_{\text {bits }}=10$ bits. For the surface height measurements, the exposure times are in the order of $1 \mathrm{~ms}$ which yields dark current noise almost at $5 \times 10^{-7} \mathrm{e}^{-} /$pixel. This confirms that dark current noise is negligible. One then plots $\sigma_{h}$ for the ideal case $\left(m_{1}=m_{2}=m\right.$ and $\left.\alpha_{1}=\alpha_{2}=\alpha\right)$, noted $\sigma_{h, \text { ideal }}$, 
as a function of the synthetic wavelength for two values of the roughness parameter $S_{q}$ at $5 \mu \mathrm{m}$ and $20 \mu \mathrm{m}$. In the case where the technical noise could be neglected, Eq. (8) would be restricted to:

$$
\sigma_{h}=\frac{\Lambda}{4 \pi}\left(\frac{7}{4}\right)\left(1-\exp \left(-8 \frac{\pi^{2}}{\Lambda^{2}} S_{q}^{2}\right)\right)^{\frac{2}{5}}
$$

and Eq. (8) can be limited to:

$$
\sigma_{h} \approx \frac{7}{16 \pi} \Lambda^{1 / 5}\left(8 \pi^{2} S_{q}^{2}\right)^{2 / 5}
$$

Figure 2 and Fig. 3 show the curves for the noise standard deviations with $\lambda_{1}=0.6328 \mu \mathrm{m}$. The curves in Figs. 2,3 have two y-axes. The left axis corresponds to the standard deviation of the surface height. The limitation of $\sigma_{h, \text { ideal }}$ (Eq. (9)), noted $D L$, is calculated by assuming that the noise related to the acquisition (photons, quantization, $\mathrm{RoN}, \ldots)$ is irrelevant compared to speckle noise $\left(\sigma_{\epsilon}\right)$. The standard deviation is multiplied by 10 to be observable. The curve allows explaining the evolution with power $1 / 5$ of Eq. (9) from Eq. (8). The latter is the product of two factors where the synthetic wavelength appears. The decay of the second factor, the phase noise term related to speckle, decreases more slowly than the increase of the first factor. Figures 2,3 show that the deviation between the exact relationship and the limited expression of the noise standard deviation decreases as the roughness increases. This is because the instrumental noise of the sensor becomes irrelevant compared to the contribution of the speckle decorrelation from roughness.

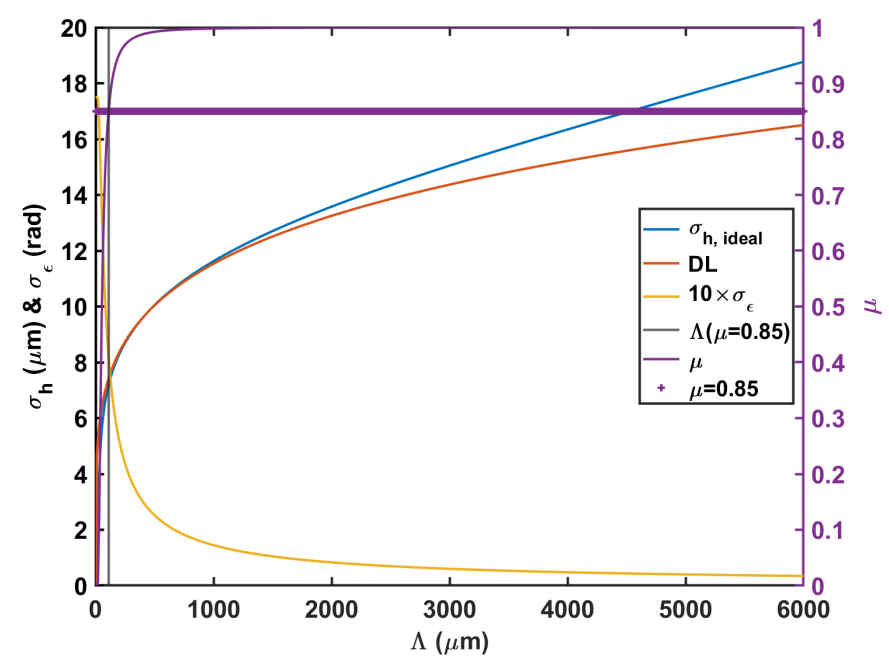

Figure 2. Standard deviation of surface height noise as function of synthetic wavelength for $S_{q}=5 \mu \mathrm{m}$. The standard deviation of decorrelation noise is plotted with a scale factor of 10 for better visibility. The second vertical axis (on the right) corresponds to the values of $|\boldsymbol{\mu}|$ and the criterion $|\boldsymbol{\mu}|=0.85$.

The tolerance criterion proposed by Poittevin ${ }^{17}$ states $|\boldsymbol{\mu}|=0.85$ and imposes a minimum synthetic wavelength that depends on $S_{q}$ such that $\Lambda_{m i n} \geq \sqrt{-8 \pi^{2} S_{q}^{2} / \ln (0.85)}$. A second criterion consists in considering the dynamics of $\Lambda / 2$ to avoid phase jumps in the measurement. But this second criterion can be in contradiction with the first one. The best choice is therefore to consider the synthetic wavelength which verifies both criteria. However, an increase in the synthetic wavelength will result in an increase in noise on the height measurement as shown in Figs 2,3. Finally, any deviation from the ideal case, i.e., $\alpha_{1}, \alpha_{2}<0.25$ and $m_{1}, m_{2}<1$, results in an increase in measurement uncertainty. 


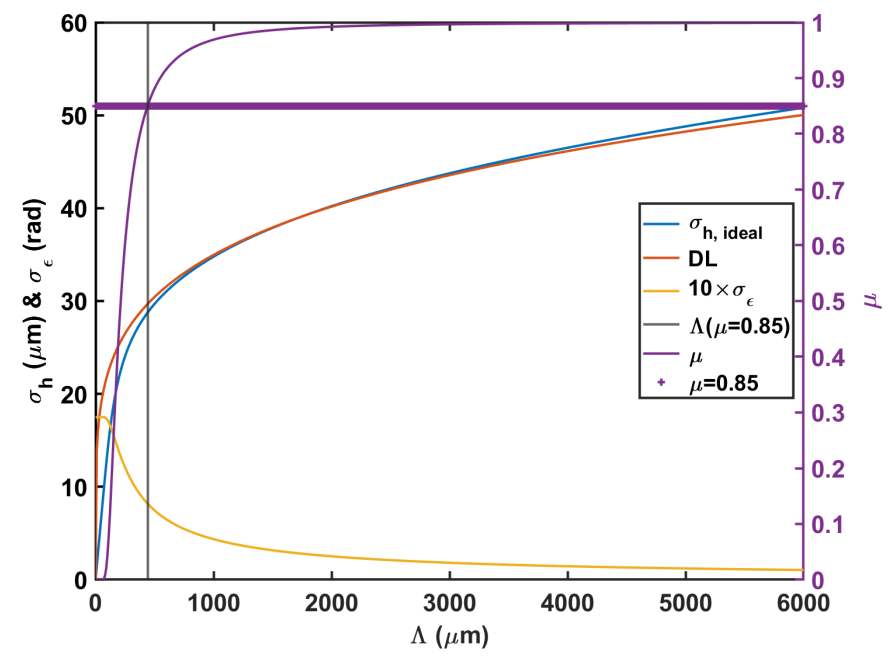

Figure 3. Standard deviation of surface height noise as function of synthetic wavelength for $S_{q}=20 \mu \mathrm{m}$. The standard deviation of decorrelation noise is plotted with a scale factor of 10 for better visibility. The second vertical axis (on the right) corresponds to the values of $|\boldsymbol{\mu}|$ and the criterion $|\boldsymbol{\mu}|=0.85$.

\section{CONCLUSION}

This paper proposes the theoretical analysis of the standard deviation of noise in the surface-height data from two-wavelength spatially-multiplexed digital holograms. The influence of noise on the measurements of the surface shape is described by an analytical approach. Relationships to quantify the minimum measurable surface height is given by taking into account the experimental parameters of the set-up. These parameters are related to the spatial bandwidths, modulation of holograms, saturation ratio, number of electrons in pixels, readout noise, quantization noise, photon noise, and speckle decorrelation due to roughness. The theoretical modeling can be used when considering practical situation for surface shape measurements.

\section{ACKNOWLEDGMENTS}

This work has been carried out in the framework of a PhD Thesis program supported by ONERA, the French Aerospace Lab, and Direction Générale de l'Armement (DGA).

\section{REFERENCES}

[1] Wyant, J. C., Oreb, B. F., and Hariharan, P., "Testing aspherics using two-wavelength holography: use of digital electronic techniques," Applied Optics 23(22), 4020-4023 (1984).

[2] Ninane, N. and Georges, M. P., "Holographic interferometry using two-wavelength holography for the measurement of large deformations," Applied Optics 34(11), 1923-1928 (1995).

[3] Pedrini, G., Fröning, P., Tiziani, H. J., and Mendoza Santoyo, F., "Shape measurement of microscopic structures using digital holograms," Optics Communications 164(4-6), 257-268 (1999).

[4] Lehmann, P., "Surface-roughness measurement based on the intensity correlation function of scattered light under speckle-pattern illumination," Applied Optics 38(7), 1144-1152 (1999).

[5] Picart, P., [New techniques in digital holography], John Wiley \& Sons (2015).

[6] Kolenovic, E., Osten, W., Klattenhoff, R., Lai, S., Kopylow, C. v., and Jüptner, W., "Miniaturized digital holography sensor for distal three-dimensional endoscopy," Applied Optics 42(25), 5167-5172 (2003).

[7] Kandulla, J., Kemper, B., Knoche, S., and von Bally, G., "Two-wavelength method for endoscopic shape measurement by spatial phase-shifting speckle-interferometry," Applied Optics 43(29), 5429-5437 (2004). 
[8] Khodadad, D., Bergström, P., Hällstig, E., and Sjödahl, M., "Fast and robust automatic calibration for single-shot dual-wavelength digital holography based on speckle displacements," Applied Optics 54(16), 5003-5010 (2015).

[9] Pedrini, G., Alekseenko, I., Jagannathan, G., Kempenaars, M., Vayakis, G., and Osten, W., "Feasibility study of digital holography for erosion measurements under extreme environmental conditions inside the International Thermonuclear Experimental Reactor tokamak," Applied Optics 58(5), A147-A155 (2019).

[10] Fratz, M., Beckmann, T., Anders, J., Bertz, A., Bayer, M., Gießler, T., Nemeth, C., and Carl, D., "Inline application of digital holography," Applied Optics 58(34), G120-G126 (2019).

[11] Kreis, T., [Handbook of Holographic Interferometry: Optical and Digital Methods], Wiley, 1st ed. (2004).

[12] Goodman, J. W., [Speckle phenomena in optics: theory and applications], Roberts, Englewood (2007).

[13] Picart, P., Montresor, S., Sakharuk, O., and Muravsky, L., "Refocus criterion based on maximization of the coherence factor in digital three-wavelength holographic interferometry," Optics Letters 42(2), 275-278 (2017).

[14] Yamaguchi, I., Yamamoto, A., and Kuwamura, S., "Speckle decorrelation in surface profilometry by wavelength scanning interferometry," Applied Optics 37(28), 6721-6728 (1998).

[15] Fercher, A. F. and Vry, U., "Two-wavelength speckle interferometric technique for rough surface contour measurement," Optical Engineering 25(5), 623-626 (1986).

[16] Gong, L., Penelet, G., and Picart, P., "Noise and bias in off-axis digital holography for measurements in acoustic waveguides," Applied Optics 60(4), A93-A103 (2021).

[17] Poittevin, J., Picart, P., Gautier, F., and Pezerat, C., "Quality assessment of combined quantizationshot-noise-induced decorrelation noise in high-speed digital holographic metrology," Optics Express 23(24), 30917-30932 (2015). 